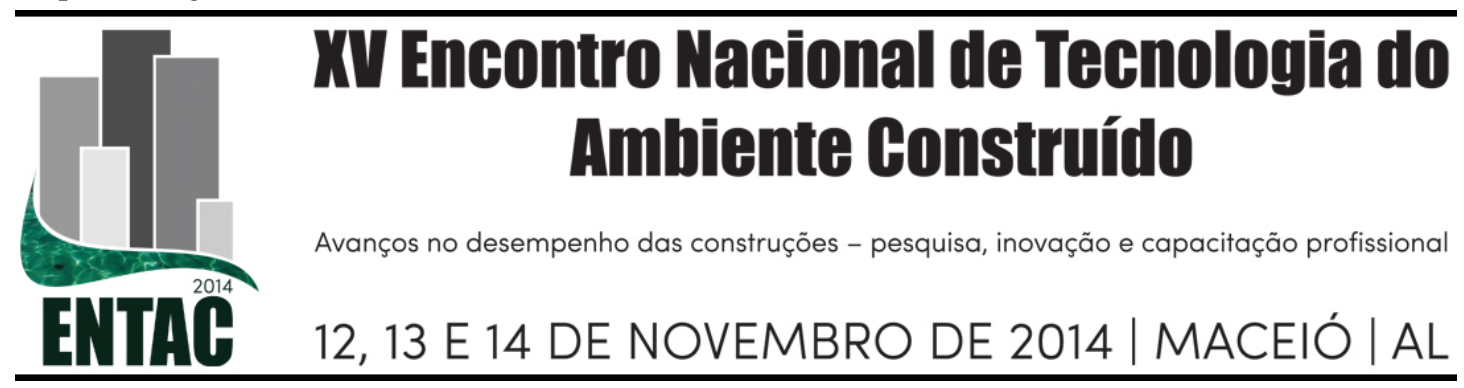

\title{
PROJETO DE SISTEMAS PREDIAIS HIDRÁULICOS SANITÁRIOS COM BIM: MAPEAMENTO DA LITERATURA
}

COSTA, Carolina H. A. (1); STAUT, Solange L. S. (2); ILHA, Marina S. O. (3)

(1) FEC/Unicamp, (19) 35212995, carolina@fec.unicamp.br (2) FEC/Unicamp, lisegle@fec.unicamp.br

(3) FEC/Unicamp, milha@fec.unicamp.br

\begin{abstract}
RESUMO
Building Information Modeling (BIM) vem sendo cada vez mais utilizado no projeto de edifícios, trazendo benefícios a todo seu ciclo de vida. Contudo, verifica-se que o uso desse conceito no projeto dos Sistemas Prediais Hidráulicos Sanitários (SPHS) no Brasil está defasado em relação às demais disciplinas: poucos são os escritórios que desenvolvem o projeto dos SPHS com o uso de BIM e praticamente não existem pesquisas desenvolvidas sobre este tema no país. Este trabalho tem como objetivo levantar as pesquisas desenvolvidas nesse tema, identificar as razões dessa defasagem e problemas que projetistas de outras especialidades e de outros países tiveram na implantação do BIM, subsidiando o desenvolvimento de trabalhos científicos que contribuam para a disseminação da ferramenta no projeto desses sistemas. Para tanto, foi desenvolvido um Sistematic Mapping Study (SMS), uma das ferramentas do paradigma baseado em evidências. A partir da seleção de expressões-chave, foram realizadas buscas de artigos de periódicos e anais de eventos nas bases de dados Scopus, Engineering Village, Web of Science e ProQuest. Em uma segunda fase, foram levantados os artigos publicados em Ambiente Construído, Gestão e Tecnologia do Projeto e InfoHab. Não foram encontradas publicações sobre SPHS especificamente, sendo que a maior parte das pesquisas recentes aponta como principais dificuldades na implantação de BIM questões ligadas à organização e dinâmica do projeto, necessidade de ferramentas de desenvolvimento como softwares e bibliotecas, e a necessidade de melhoria na interoperabilidade entre ferramentas.
\end{abstract}

Palavras-chave: Building Information Modeling, Systematic Literature Review, Sistemas Prediais Hidráulicos Sanitários.

\begin{abstract}
Building Information Modeling (BIM) is being increasingly used in building design, providing benefits to the entire lifecycle. However, the use of BIM in Plumbing Systems design in Brazil is outdated compared to other disciplines: there are few offices that develop Plumbing Systems design using BIM, and research development on this subject in Brazil is incipient. This study aims to gather research under this subject and identify the reasons for the lag and issues that designers from other specialties and other countries have had in deploying BIM, supporting scientific work to contribute to the use of this tool in brazilian offices. To this end, it was developed a Systematic Mapping Study (SMS), an evidence-based paradigm tool. From the selection of key expressions, journal articles and conference proceedings were searched in Scopus, Engineering Village, Web of Science and ProQuest. In a second phase, a new search was performed in national basis Ambiente Construído, Gestão e Tecnologia do Projeto e InfoHab. The results contribute to identify gaps in the research development in Plumbing Systems design using BIM. There were not found researches in this subject. The recent researches show that the main barriers in implementing BIM are related to project organization and dynamics, the need for tools development such as software and libraries, and interoperability.
\end{abstract}

Keywords: Building Information Modeling, Systematic Literature Review, Plumbing Systems. 


\section{INTRODUÇÃO}

O desempenho das edificações é fortemente determinado por decisões tomadas na fase de projeto. O uso de modelos em um ambiente virtual permite, por exemplo, a avaliação da solução mais econômica e sustentável para cada decisão a ser tomada desde a concepção inicial de um projeto.

BIM é mais do que um modelo de visualização do espaço projetado. Consiste em um modelo digital composto por uma base de dados que transmite mais informação do que os modelos CAD (Computer Aided Design) tradicionais, preparando modelos de edifício dentro de um ambiente virtual em qualquer escala, podendo ser automaticamente detalhados e analisados (COELHO E NOVAES, 2008).

Segundo Eastman et al. (2014), o projeto é a atividade em que a maior parte das informações sobre um empreendimento é definida e em que a estrutura é organizada para posterior detalhamento. Devido à sua capacidade de automatização de detalhamento, o BIM reduz o tempo necessário para a produção de documentos para a construção, já que as informações são inseridas no projeto na fase de concepção, facilitando a interação de diferentes ferramentas e melhorando a disponibilidade de informação para outros agentes. Para o bom andamento do processo mostra-se fundamental garantir a colaboração entre todos os intervenientes em todas as fases de desenvolvimento do edifício: concepção, construção e operação.

Verifica-se um crescente uso de BIM no projeto arquitetônico e de estruturas no Brasil, contudo, isso não acontece no desenvolvimento do projeto dos Sistemas Prediais Hidráulicos Sanitários (SPHS), sendo poucos os escritórios que empregam BIM no projeto dos SPHS e praticamente não existem pesquisas nesse tema no país.

Com o objetivo de caracterizar o estágio de desenvolvimento das pesquisas sobre BIM aplicado ao projeto dos SPHS em outros países e no Brasil e identificar lacunas do conhecimento nesse tema, foi desenvolvido um mapeamento sistemático da literatura (SMS), conforme proposto por Kitchenham et al. (2010).

Além de auxiliar na identificação de lacunas do conhecimento, segundo Ruiz e Granja (2013), o SMS permite a identificação de áreas de estudos realizados (MELO, GRANJA e BALLARD, 2013) e contribui com a comunidade acadêmica por possibilitar tanto a continuidade de trabalhos já publicados como o surgimento de propostas realmente inovadoras. Isso decorre do fato de que o SMS oferece uma visão geral do tema pesquisado, permitindo a identificação de quantidade, tipo e resultados já obtidos com os estudos encontrados.

\section{FUNDAMENTAÇÃO}

Succar (2009) define BIM como sendo um conjunto de políticas, processos e tecnologias que interagem originando uma metodologia que gerencia o projeto de construção e seus dados em formato digital ao longo do ciclo de vida do edifício. Segundo Eastman et al. (2014), o BIM opera como uma simulação inteligente da arquitetura, e para que seja integrada, esta deve ser digital, espacial (3D), mensurável, abrangente, acessível e utilizável ao longo de todo o ciclo de vida da edificação.

Ainda de acordo com Eastman et al. (2014) à medida que o modelo de construção pode ser produzido por uma ferramenta BIM dando suporte aos vários dados contidos dentro de um conjunto de desenhos (2D e 3D), esse modelo pode ser caracterizado por quais objetos descreve e quais requisitos de informação ele atende.

Korman e Lu (2011) afirmam que os conflitos físicos entre elementos estruturais, elétricos ou hidráulicos podem ser facilmente detectados com os modelos virtuais e que 
o uso destes no processo de compatibilização é muito importante, especialmente no caso dos sistemas prediais.

O principal atrativo do uso de BIM, entretanto, não está na fase de projeto propriamente dita, mas na quantidade e qualidade de informações disponíveis no mesmo, que gerará economias substanciais nas próximas etapas, além de permitir ao projetista a oferta de novos serviços tais como programação e estimativa de custos, listas de materiais, serviços pré e pós-construção, detecção de interferências e gestão de facilidades.

Com a crescente complexidade dos edifícios, é natural que a equipe envolvida no projeto seja mais numerosa e especializada, o que requer um processo de comunicação, colaboração e coordenação mais sofisticado. Atualmente os sistemas de climatização, elétrica, hidráulica e combate a incêndio podem representar até $40 \%$ do escopo de um edifício. Assim, esses agentes precisam colaborar não apenas entre si, mas também com responsáveis pela arquitetura, estrutura e demais disciplinas envolvidas. Contudo, apesar da tecnologia possibilitar a colaboração, as empresas apresentam questões organizacionais que limitam a interação, independentemente de empregar BIM ou não (DOSSICK e NEFF, 2010).

Apesar das vantagens associadas ao uso de BIM, verifica-se que o emprego dessa ferramenta no desenvolvimento do projeto dos SPHS é ainda tímido. Com este mapeamento da literatura pretende-se identificar os principais avanços obtidos pelas pesquisas realizadas e as lacunas no conhecimento, de modo a subsidiar o desenvolvimento de pesquisas futuras sobre esse tema.

\section{MÉTODO}

As etapas envolvidas no mapeamento sistemático da literatura são apresentadas na Figura 1, tendo por base o método empregado por Ruiz e Granja (2013) e Melo, Granja e Ballard (2013), a partir do método proposto por Kitchenham (2010).

Figura 1 - Desenvolvimento do Mapeamento Sistemático da Literatura (SMS)

\begin{tabular}{|c|c|c|c|c|}
\hline \multicolumn{2}{|c|}{ PLANEJAMENTO } & \multicolumn{2}{|c|}{ CONDUÇÃO } & DOCUMENTA \\
\hline \begin{tabular}{|c|} 
DEFINIČ̃A DO \\
TEMA
\end{tabular} & $\begin{array}{c}\text { SELEÇÃO DA BASE DE } \\
\text { DADOS }\end{array}$ & $\begin{array}{c}\text { CRITÉRIOS DE SELEÇÃO } \\
\text { DOS ARTIGOS }\end{array}$ & $\begin{array}{c}\text { EXTRAČ̃̃O } \\
\text { SISTEMATIZADA DEDADOS }\end{array}$ & $\begin{array}{l}\text { SÍNTESE DE } \\
\text { DADOS }\end{array}$ \\
\hline & & & & 15 \\
\hline & $\begin{array}{c}\text { FORMULAÇÃODA STRING } \\
\text { DEBUSCA }\end{array}$ & & $\begin{array}{c}\text { AMOSTRAGEM EM } \\
\text { SNOWBALL }\end{array}$ & $\begin{array}{l}\text { ANÁLISE DE } \\
\text { DADOS }\end{array}$ \\
\hline & & & CODIFICAÇÃO DEDADOS & \\
\hline
\end{tabular}

Fonte: Adaptado de Kitchenham (2010), Ruiz e Granja (2013) e Melo, Granja e Ballard (2013).

As bases de dados internacionais selecionadas foram: Scopus, Web of Science, Engineering Village (Compendex) e ProQuest, sendo que todas elas possibilitam o uso de strings de busca complexas, com o emprego de operadores booleanos.

A string de busca nas bases internacionais foi definida tendo em vista as questões:

- Qual o estágio do desenvolvimento das pesquisas relacionadas ao uso de BIM nas diferentes disciplinas de projeto, com destaque aos SPHS?

- Quais os principais enfoques e lacunas das pesquisas desenvolvidas com o uso de BIM nas diferentes disciplinas de projeto, com destaque aos SPHS? 
A partir dessas questões de pesquisa, foram selecionados os seguintes termos: BIM, building information modelling, design, Project, MEP, plumbing, water supply, building system, construction, building.

Resultando na seguinte string de busca: (BIM OR "Building Information Model*") AND (design* OR project*) AND (mep OR plumb* OR "Water Supply" OR "Building System*" OR building OR construction).

Nota: MEP - Mechanical, Electrical and Plumbing, que contemplam os SPHS.

No caso da busca nas bases internacionais, foram adotados os seguintes critérios: string constante no título, resumo ou palavras-chave; somente artigos de periódicos e de anais de eventos e somente artigos publicados em inglês ou português.

Inesperadamente, a pesquisa trouxe como resultado artigos de outras áreas do conhecimento, como medicina, artes e administração.

A partir da remoção dos artigos de outras áreas e efetuada a análise conjunta dos resultados obtidos em todas as bases de dados internacionais, de modo a eliminar artigos repetidos, obteve-se o número de artigos final.

Com a leitura dos títulos dos artigos, foram identificadas palavras-chave de interesse. $\mathrm{O}$ processo foi efetuado de modo iterativo: conforme um título de interesse era identificado, novas palavras-chave eram selecionadas, tendo sempre em vista as questões de pesquisa. Por fim, utilizando a ferramenta de filtro em uma planilha do Microsoft Excel, foram selecionados apenas os artigos que contivessem pelo menos uma das palavras-chave escolhidas no título ou no resumo, conforme segue: (A) no título: MEP, hidr, pipe, wate, net, libr, office, project, plumb, issue, diff, problem, implement, challeng; (B) no resumo: MEP, hidr, pip, water, library, plumb.

De posse dos artigos selecionados nas bases de dados internacionais, foi efetuada a análise detalhada dos títulos e resumos, resultando em um novo conjunto de artigos, os quais foram lidos em sua totalidade, levando à exclusão de mais alguns. A partir disso, obteve-se o número final de artigos a ser considerado nas demais etapas da pesquisa. Destaca-se que nessa fase foram considerados somente os artigos com texto completo e com acesso gratuito via biblioteca virtual da universidade.

No caso dos artigos nacionais, a busca foi efetuada na base de dados Centro de Referência e Informação em Habitação - InfoHab e em dois periódicos nacionais: Ambiente Construído e Gestão e Tecnologia de Projetos, ambos da Associação Nacional de Tecnologia do Ambiente Construído - ANTAC. Nesses casos não existe a possibilidade de emprego de strings, de modo que o procedimento para a busca e seleção dos artigos baseou-se apenas no uso da palavra-chave BIM. De posse de todos os artigos selecionados, foi efetuada a leitura do título e do resumo, e posteriormente do texto completo, identificando-se os artigos com aderência às questões de pesquisa, resultando no número final de artigos constantes na base e nos periódicos nacionais para as demais etapas da pesquisa. Destaca-se que, a exemplo das bases internacionais, foram considerados somente os artigos com texto completo e com acesso gratuito via biblioteca virtual da universidade.

Diante da possibilidade de que alguns autores tenham utilizado sinônimos dos termos empregados na string de busca, o que faria com que seus artigos não fossem selecionados na pesquisa efetuada, foi desenvolvida uma pesquisa adicional denominada "bola de neve" (snowball sampling). Assim, a partir da análise da lista de referências de cada artigo selecionado, foram selecionados artigos (apenas de periódicos) com conteúdo aderente ao tema e que não constavam no conjunto de artigos já selecionados, conforme Melo, Granja e Ballard (2013). 
Os artigos selecionados a partir das etapas anteriores foram classificados de duas formas:

- Disciplina de projeto: geral (sem se limitar a uma disciplina específica), arquitetura, estrutura e sistemas prediais (mecânicos, elétricos e hidráulicos);

- Principal contribuição (categoria), conforme a Tabela 1.

Tabela 1 - Categorias para classificação dos artigos das bases internacionais.

\begin{tabular}{l|l}
\hline \multicolumn{1}{c|}{ Categoria } & \multicolumn{1}{c}{ Artigos que contemplam ... } \\
\hline Conceitos & $\begin{array}{l}\text { a apresentação do conceito do BIM de forma abrangente, sem focar em } \\
\text { problema específico. }\end{array}$ \\
\hline Ferramentas & $\begin{array}{l}\text { o uso de softwares, padrões, bibliotecas e outros elementos que apoiam } \\
\text { o projeto. }\end{array}$ \\
\hline Processo de Projeto & $\begin{array}{l}\text { a análise das principais mudanças que o BIM trouxe para a dinâmica do } \\
\text { projeto e da equipe. }\end{array}$ \\
\hline Coordenação & $\begin{array}{l}\text { o novo papel do profissional da coordenação e o processo de } \\
\text { compatibilização. }\end{array}$ \\
\hline Análise econômica & a análise de custos e de ganhos com a aplicação de BIM. \\
\hline Papel do Governo & $\begin{array}{l}\text { padronizações e contrapartidas oferecidas por governos que exigem o } \\
\text { uso de BIM na contratação dos seus projetos. }\end{array}$ \\
\hline
\end{tabular}

Além disso, foi efetuada a classificação dos artigos especificamente de sistemas prediais, conforme as categorias apresentadas na Tabela 2Tabela 3.

Tabela 2 - Categorias para classificação dos artigos de sistemas prediais.

\begin{tabular}{l|l}
\hline \multicolumn{1}{c|}{ Categoria } & \multicolumn{1}{c}{ Artigos que contemplam ... } \\
\hline Simulação & $\begin{array}{l}\text { a apresentação das vantagens de usar ferramentas de simulação para a } \\
\text { concepça do edifício. }\end{array}$ \\
\hline Pré-fabricação & $\begin{array}{l}\text { o uso de BIM para gerar elementos para a obra, melhorando a } \\
\text { produtividade. }\end{array}$ \\
\hline Compatibilização & o processo de compatibilização com BIM. \\
\hline $\begin{array}{l}\text { Implantação em } \\
\text { escritórios }\end{array}$ & orientações para o processo de implantação de BIM em escritórios. \\
\hline
\end{tabular}

Por fim, foram identificadas as lacunas ou temas para o desenvolvimento de pesquisas futuras apontadas nos diferentes artigos.

\section{RESULTADOS E DISCUSSÃO}

A Tabela 3 e a Tabela 4 apresentam os resultados da busca efetuada nas bases de dados internacionais. Não se teve acesso gratuito ao texto completo de 12 artigos via biblioteca virtual da universidade, por isso estes textos foram descartados. 
Tabela 3 - Número de artigos encontrados em cada base de dados internacional.

\begin{tabular}{l|c}
\hline \multicolumn{1}{c|}{ Base de dados } & Número de artigos \\
\hline Scopus & 1484 \\
\hline Web of Science & 478 \\
\hline Engineering Village & 1333 \\
\hline ProQuest & 2515 \\
\hline
\end{tabular}

Tabela 4 - Resultado da busca nas bases de dados internacionais.

\begin{tabular}{l|c}
\hline \multicolumn{1}{c|}{ Atividade } & $\begin{array}{c}\text { Núm. de } \\
\text { artigos }\end{array}$ \\
\hline encontrados em todas as bases & 5810 \\
\hline encontrados em todas as bases (sem os artigos repetidos e de outras áreas) & 2221 \\
\hline selecionados após o uso do filtro no título e resumo & 362 \\
\hline selecionados após a leitura do título e do resumo & 41 \\
\hline selecionados após a leitura do título e resumo e com acesso ao texto completo & 29 \\
\hline selecionados após a leitura do texto completo & 20 \\
\hline final selecionado após a snowball sampling & 21 \\
\hline
\end{tabular}

Os Estados Unidos e o Reino Unido concentram a maioria dos trabalhos dentro do tema em estudo (Figura 2). Trata-se de um assunto recente, com publicações a partir de 2008.

Figura 2 - Distribuição dos artigos selecionados nas bases de dados internacionais em função do ano de publicação e do país da instituição do primeiro autor.

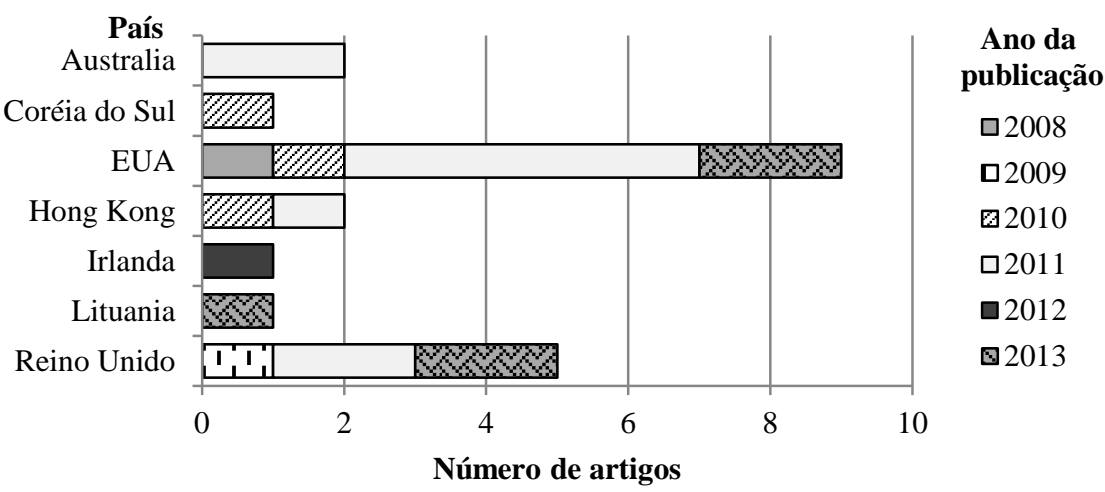

Figura 3 - Distribuição dos artigos selecionados nas bases de dados internacionais nas categorias identificadas e em função do ano de publicação.

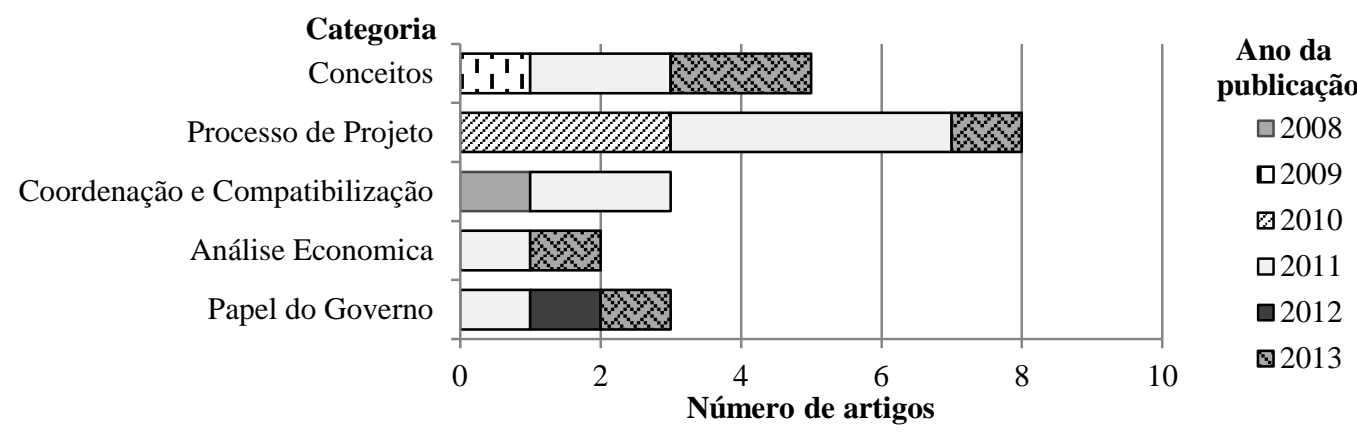


O processo do projeto é o tema mais abordado, seguido da apresentação de conceitos, com 38\% e 24\% dos artigos selecionados, respectivamente (Figura 3).

A maioria dos artigos contempla o uso de BIM às disciplinas do projeto como um todo, (Figura 4). Considerando-se apenas os 4 artigos que contemplam os sistemas prediais, foram em sua totalidade desenvolvidos nos Estados Unidos e verifica-se que não há uma predominância de tema abordado (Figura 5).

Figura 4 - Distribuição dos artigos selecionados nas bases de dados internacionais dentro das disciplinas de projeto em função do ano de publicação.

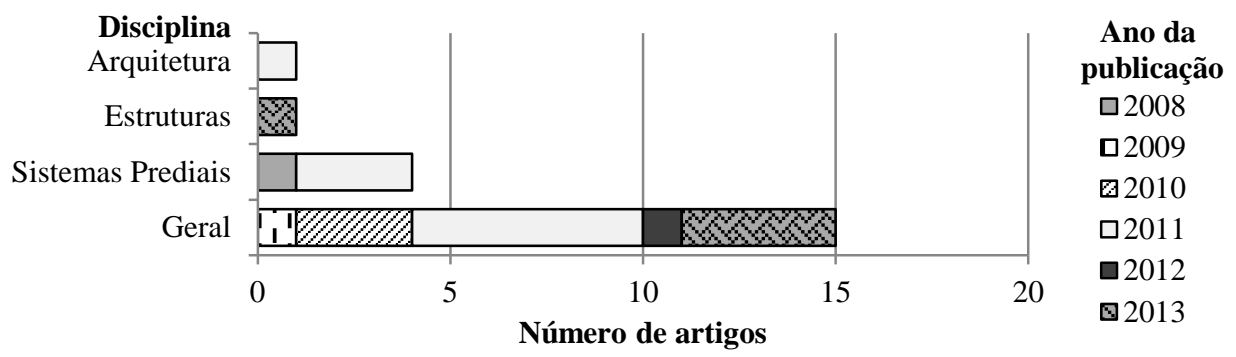

Figura 5 - Distribuição dos artigos selecionados nas bases relacionados com sistemas prediais nas categorias identificadas e em função do ano de publicação.

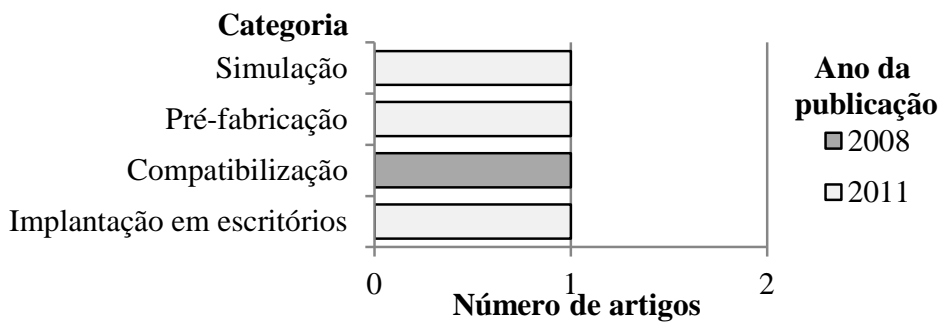

As principais lacunas de pesquisa apontadas nos artigos selecionados são apresentadas nas Figura 6 e Figura 7. A interoperabilidade (possibilidade de se utilizar um mesmo modelo virtual pelos projetistas das diferentes disciplinas e seus respectivos softwares) e o desenvolvimento de ferramentas, tais como bibliotecas, softwares de simulação e padrões em geral são as principais lacunas de pesquisa, apontadas em artigos de praticamente todas as categorias.

Figura 6 - Lacunas de pesquisa apontadas nos artigos selecionados nas bases de dados internacionais por categoria, independentemente da disciplina de projeto.

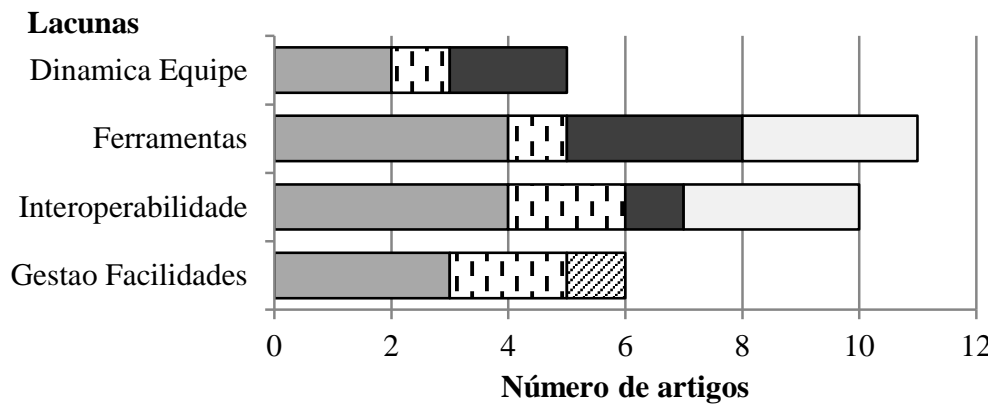

\footnotetext{
Categoria

口Conceitos

口Processo de Projeto

๑Coordenação e

Compatibilização

๒ Análise Economica

口Papel do Governo
} 
Figura 7 - Lacunas de pesquisa apontadas nos artigos selecionados nas bases de dados internacionais por categoria, apenas artigos de sistemas prediais

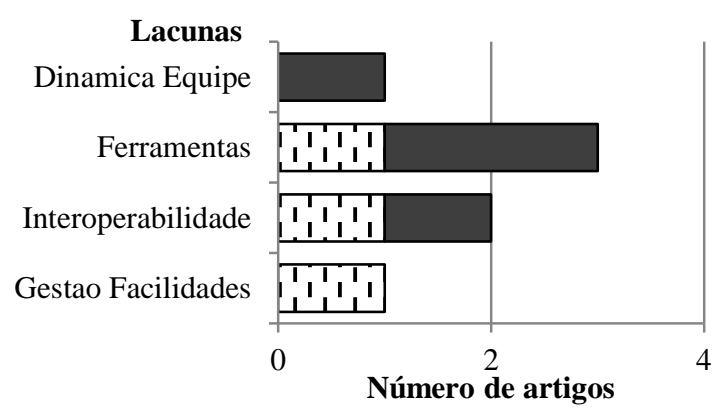

\author{
Categoria \\ 口Processo de Projeto \\ ๑Coordenação e \\ Compatibilização
}

A Tabela 5 e a Tabela 6 apresentam os resultados da busca na base de dados e nos periódicos nacionais. Não se teve acesso gratuito ao texto completo de 6 artigos via biblioteca virtual da universidade, por isso estes textos foram descartados. A distribuição dos artigos é apresentada na Tabela 5.

Tabela 5 - Número de artigos encontrados nas fontes nacionais.

\begin{tabular}{l|c}
\hline Fonte & Número de Artigos \\
\hline Base de dados InfoHab & 22 \\
\hline Periódico Ambiente Construído & 7 \\
\hline Periódico Gestão e Tecnologia de Projeto & 11 \\
\hline
\end{tabular}

Tabela 6 - Resultado da busca nas fontes nacionais.

\begin{tabular}{l|c}
\hline \multicolumn{1}{c|}{ Atividade } & $\begin{array}{c}\text { Núm. de } \\
\text { artigos }\end{array}$ \\
\hline encontrados em todas as fontes nacionais & 40 \\
\hline encontrados em todas as fontes (sem artigos repetidos e de outras áreas) & 35 \\
\hline selecionados após a leitura do título e do resumo & 19 \\
\hline selecionados após a leitura do título e do resumo e com texto completo & 13 \\
\hline selecionados após a leitura do texto completo & 9 \\
\hline final selecionado após a snowball sampling & 10 \\
\hline
\end{tabular}

Verifica-se que as pesquisas estão mais concentradas no estado de São Paulo. Não foram localizados artigos relacionados com sistemas prediais; sendo que a maioria trata o tema de forma geral ou apenas a disciplina de Arquitetura. Não foi identificada nenhuma categoria prevalecente dentre os artigos analisados (Figura 8).

A maioria dos artigos levantados nas fontes nacionais aponta o desenvolvimento de ferramentas como uma lacuna de pesquisa, seguido da dinâmica da equipe de projeto como um todo (Figura 9). A interoperabilidade é uma lacuna de pesquisa pouco citada, o que se deve justamente à falta de ferramentas disponibilizadas para o projeto. 
Figura 8 - Distribuição dos artigos selecionados nas fontes nacionais em função: (a) do ano de publicação e da instituição do primeiro autor; (b) da disciplina de projeto e (c) da categoria.

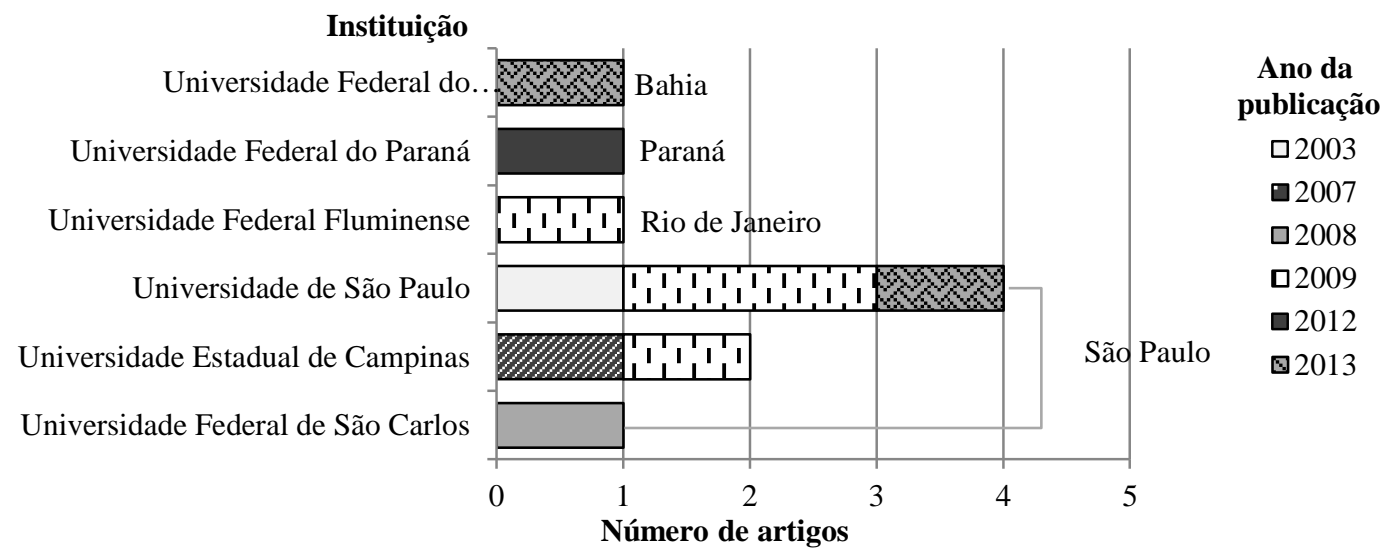

(a)

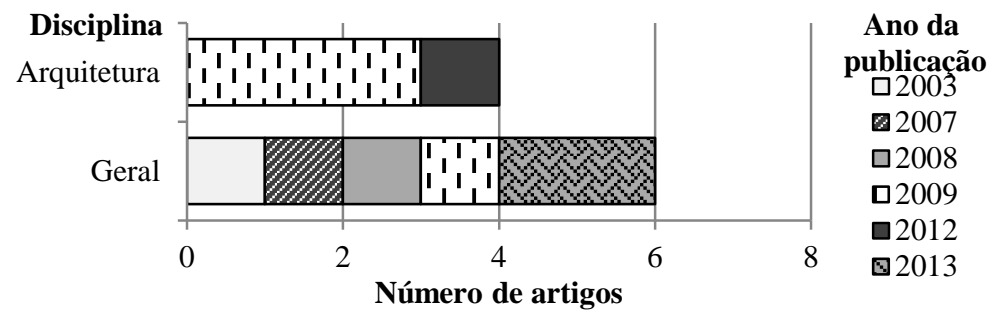

(b)

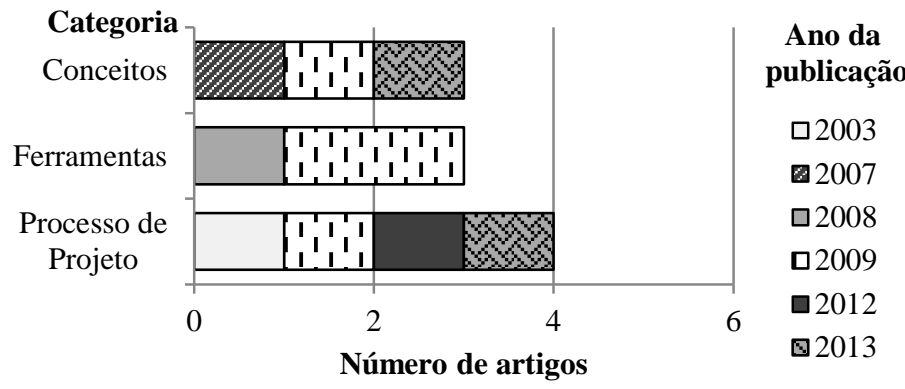

(c)

Figura 9 - Lacunas de pesquisa apontadas nos artigos levantados nas fontes nacionais por categoria.

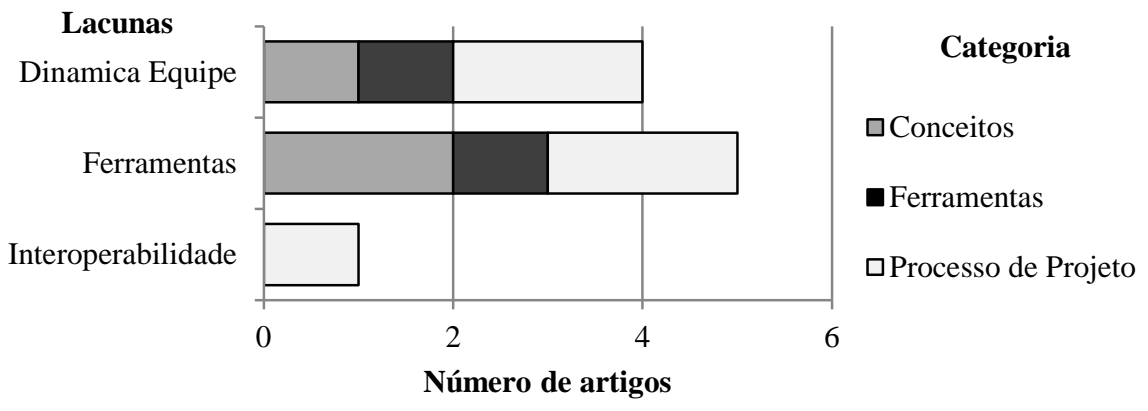




\section{CONSIDERAÇÕES FINAIS}

A partir do levantamento sistemático realizado pode-se identificar que as publicações são ainda muito voltadas para a conceituação da tecnologia, seja quando analisadas as fontes nacionais como as internacionais.

As lacunas identificadas são similares, apesar do grau de avanço das pesquisas ser menor em âmbito nacional e percebe-se que ainda há muito que ser feito.

Poucos são os exemplos de pesquisas relatadas nas bases internacionais e não foram encontrados estudos nas fontes nacionais que tratem dos sistemas prediais. Não foram encontrados estudos específicos sobre os SPHS.

Esse cenário indica a grande necessidade de desenvolvimento de pesquisas nesse tema, tendo em vista os benefícios que o BIM proporciona em outras disciplinas, ao longo de todo o ciclo de vida.

\section{AGRADECIMENTOS}

À Faculdade de Engenharia Civil, Arquitetura e Urbanismo (FEC), à Agência para Formação Profissional da Unicamp (AFPU) e à Fundação de Amparo à Pesquisa do Estado de São Paulo (FAPESP), processo nº 2014/16362-1, pelo apoio na divulgação da pesquisa.

\section{REFERÊNCIAS}

COELHO, S. S. e NOVAES, C. C.; Modelagem de Informações para Construção (BIM) e ambientes colaborativos para gestão de projetos na construção civil. In: VIII Workshop Nacional de Gestão do Processo de Projeto na Construção de Edifícios, São Paulo, Anais ...2008.

DOSSICK C.S.; NEFF G. Organizational divisions in bim-enabled commercial construction. Journal of Construction Engineering and Management. v. 136, n. 4, p. 459-467, 2010.

EASTMAN, C.; TEICHOLZ, P.; SACKS, R.; LISTON, K. Manual de BIM Um guia de Modelagem da Informação da Constrição para Arquitetos, Engenheiros, Gerentes , Construtores e Incorporadores. Bookman, Porto Alegre: 2014. 483 p.

KITCHENHAM B.;PRETORIUS R.; BUDGEN D.; BRERETON O. P.; TURNER M.; NIAZI.; LINKMAN S.; Systematic literature reviews in software engineering - A tertiary study Review.Information and Software Technology, v. 52, 2010, p. 792-805

KORMAN T.M.; LU N. Innovation and improvements of mechanical electrical and plumbing systems for modular construction using building information modeling. In: AEI 2011: Building Integrated Solutions, 2011. Proceedings..., 2011. p. 448-455.

MELO, R. S. S.; GRANJA, A. D.; BALLARD, G. Collaboration to extend target costing to non-multiparty contracted projects: evidence from literature. In: 21st Annual Summit of the International Group for Lean Construction, 2013, Fortaleza. Proceedings..., 2013. p. 1-10.

RUIZ, J.A.; GRANJA, A. D. Um mapeamento sistemático da literatura sobre a relação entre valor e colaboração na construção. In: VIII Simpósio Brasileiro de Gestão e Economia da Construção, 2013, Salvador. Anais..., 2013.

SUCCAR, B. Building information modelling framework: A research and delivery foundation for industry stakeholders. Automation in Construction. v 18, n. 3, p. 357-375, 2009. 